\title{
130. Responses of Vagina to Estrogen and Progesterone in Neonatally Androgenized Rats
}

\author{
By Kiyoshi TAKeWAKI \\ Zoological Institute, Faculty of Science, University of Tokyo \\ (Comm. by Yô K. OKadA, M.J.A., Sept. 13, 1965)
}

In adult female rats given injections of large doses of androgen for 30 successive days from the day of birth, the ovaries are highly reduced in size, containing a few small follicles but neither maturing follicles nor corpora lutea. In the vagina, three portions can be distinguished by the state of the epithelium, i.e. the proximal portion lined by an epithelium consisting of two layers of cells, the middle portion covered by a multilayered epithelium, and the distal portion with a hypertrophied and cornified epithelium. In these rats, hypophysectomy elicits no perceptible changes in the ovaries, and hypophysectomy followed by ovariectomy and adrenalectomy is without effect on the vagina. Therefore, it seems evident that the secretion of gonadotropins by the anterior hypophysis is permanently held in check and that changes in the vagina are independent of estrogenic stimulation and irreversible (Takewaki, 1965).

The current experiments were designed to answer three questions: (1) Are ovaries of androgenized rats stimulated to form corpora lutea by injections of gonadotropins? (2) If so, are the corpora lutea functional? (3) How do the three portions of vagina react to exogenous and endogenous steroid hormones?

Materials and Methods. Female rats from the colony maintained in this laboratory were given subcutaneous injections of testosterone propionate for 30 successive days from the day of birth, daily doses being $500 \mu \mathrm{g}$ in $0.02 \mathrm{ml}$ of sesame oil for the first 10 days, $1000 \mu \mathrm{g}$ in $0.04 \mathrm{ml}$ of oil for the middle 10 days and $2000 \mu \mathrm{g}$ in $0.08 \mathrm{ml}$ of oil for the last 10 days.

When rats reached 65-95 days of age, they were divided into 3 groups. In the first group, rats were hypophysectomized by the parapharyngeal route under pentobarbital anesthesia and adenohypophysis was autografted under the capsule of the left kidney. In the second group, rats were hypophysectomized but not given hypophysial grafts. Animals of the third group were left intact or sham-hypophysectomized.

All rats received a single subcutaneous injection of 75 I.U. of PMS (Anteron, Schering A.G., Berlin) in $0.15 \mathrm{ml}$ of $0.9 \% \mathrm{NaCl}$ solution 7 days after operation and its 50 I.U. in $0.1 \mathrm{ml}$ of saline solution 

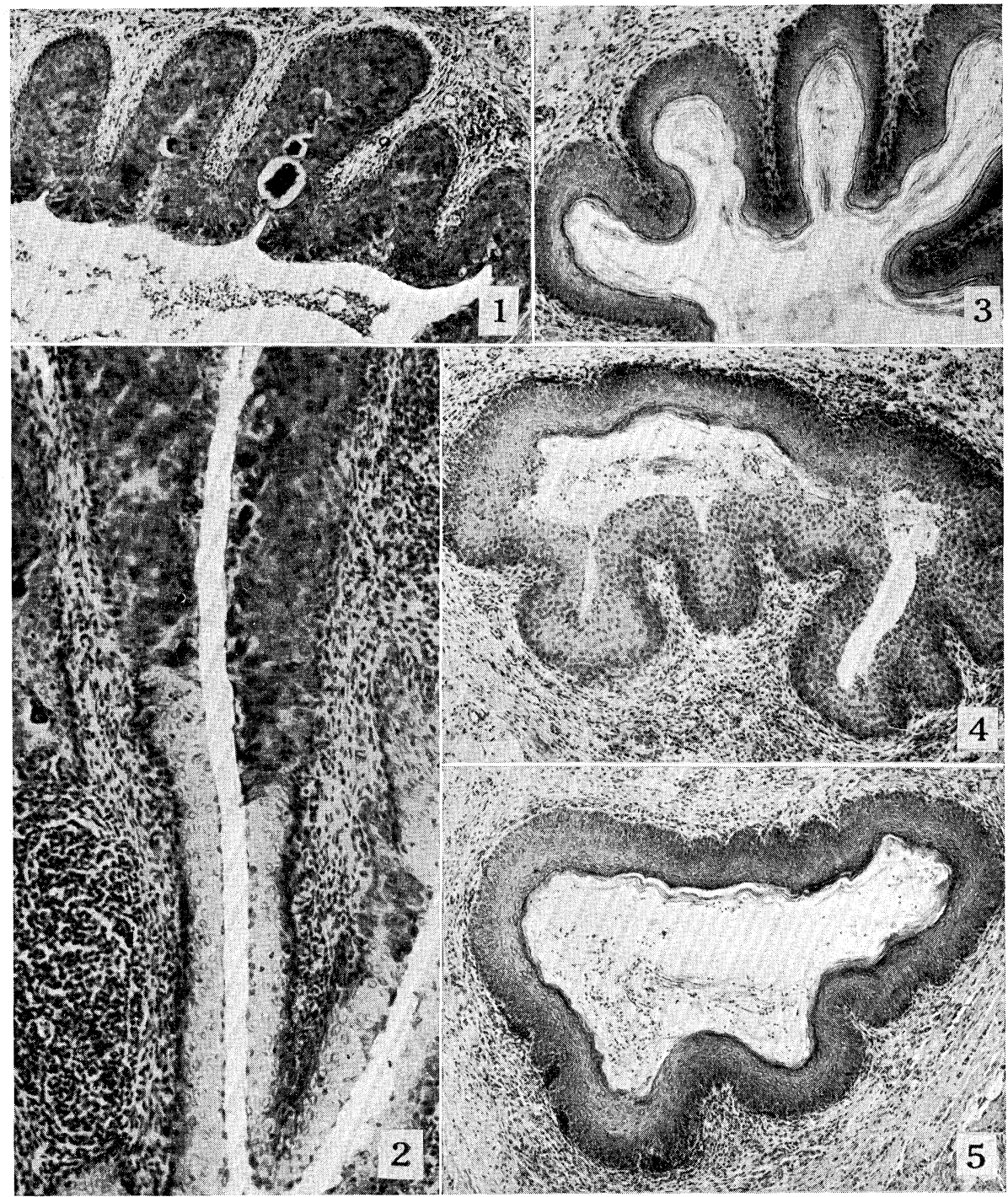

Fig. 1. Proximal portion of vagina of 107-day old hypophysectomized androgenized rat bearing intrarenal hypophysial autograft. The rat received estradiol injections after corpora lutea had been induced artificially. The epithelium is mucified. $\times 105$

Fig. 2. Transitional part between proximal and middle portions of vagina of the same rat, showing junction of two epithelia. Longitudinal section. $\times 195$

Fig. 3. Proximal portion of vagina of 95-day old intact androgenized rat. The rat received estradiol injections after corpora lutea had been induced artificially. The epithelium is cornified. $\times 105$

Fig. 4. Transitional part between proximal and middle portions of vagina of the same rat. $\times 105$

Fig. 5. Distal portion of vagina of the same rat. $\times 105$ 
for the next 2 days. Two days after the final injection of PMS, animals were given a single subcutaneous injection of 50 I.U. of HCG (Primogonyl, Schering A.G., Berlin) in $0.1 \mathrm{ml}$ of saline solution. Seven days later, they were injected subcutaneously with $10 \mu \mathrm{g}$ estradiol-17 $\beta$ in $0.1 \mathrm{ml}$ of sesame oil for 4 consecutive days, and were weighed and sacrificed on the 5th or the 6th day.

Ovaries, adrenals, uterus and vagina of each rat were fixed in Bouin's solution. Adrenals and uterus were weighed before fixation. Sections were cut in paraffin and stained with Delafield's hematoxylin and eosin.

Results of cytological studies on hypophysial grafts will be reported elsewhere in conjunction with S. Kawashima.

Results. In highly atrophic ovaries of androgenized rats, corpora lutea were induced to form by injections of gonadotropins. However, they were usually one or two per ovary, seldom counting more than three. In addition to corpora lutea, some primary and solid follicles were occasionally found in the ovaries but large vesicular follicles were rarely encountered. Interstitial tissue was not well developed.

In the proximal portion of vagina of 7 rats bearing hypophysial autografts under the renal capsule, 4 daily injections of estradiol following induced luteinization in their ovaries resulted in marked mucification of the epithelium (Fig. 1). In contrast, the epithelium of the middle portion was stratified, showing no signs of mucification or cornification (Fig. 2), and that of the distal portion remained cornified. After being subjected to similar treatments, both 10 intact (including sham-hypophysectomized) and 5 hypophysectomized animals invariably showed cornification of the epithelium of the proximal portion of vagina (Fig. 3). However, as in hypophysectomized rats bearing hypophysial autografts, the epithelia lining the middle and distal portions were unaffected, being stratified and cornified, respectively (Figs. 4, 5).

The mucified or cornified epithelium of the proximal portion of vagina was sharply delimited from the stratified epithelium of the middle portion (Figs. 2,4), suggesting that the two epithelia were different in nature.

As reported in the previous paper (1965), the vaginal orifice was not formed in the normal position in any androgenized rats. In a majority of animals, it has not been ascertained whether the distal vagina communicated with the urethra or had an orifice independent of the urethra.

Lutein cells of corpora lutea in intact and hypophysectomized rats were usually smaller and arranged more loosely than in hypo- 
physectomized rats bearing hypophysial transplants. Adrenals were decidedly smaller in hypophysectomized rats $(16.6 \pm 0.5 \mathrm{mg})$ and hypophysectomized animals with hypophysial grafts $(17.1 \pm 0.4 \mathrm{mg})$ than in those with the hypophysis intact $(48.8 \pm 2.4 \mathrm{mg})$. This finding was indicative of the completeness of hypophysectomy which was also checked by examination of the hypophysial fossae.

Results of histological studies of uteri will be dealt with in a separate paper. It may be mentioned here, however, that, in some animals, the uterine epithelium was stratified and cornified or exhibited many high branched folds.

Discussion. In rats which had received 30 daily injections of large doses of testosterone propionate from the day of birth, injections of gonadotropins caused follicular growth followed by luteinization in their ovaries. Therefore, it seems evident that marked atrophy of ovaries in such animals was due to lack of gonadotropins rather than to loss of susceptibility of the ovaries to gonadotropins.

Several workers have pointed out that a decrease in the secretion of gonadotropins is usually accompanied by an increase in the production and release of luteotropin, and vice versa. However, the results detailed above have shown that in androgenized rats the release of luteotropin is inhibited by the hypothalamus, corpora lutea artificially produced in their ovaries being activated to function only after the connection between the hypothalamus and the anterior hypophysis is severed. Similar findings in persistent-diestrous rats secured by prolonged neonatal administration of estradiol have already been reported (Takewaki, 1964). These findings are not in harmony with the concept that gonadotropin-releasing factor is identical with luteotropin-inhibiting factor but are consistent with the recent results of Pasteels (1963) and Schally, Meites, Bowers, and Ratner (1964) suggesting that the two factors are not the same.

In the vagina of androgenized rats, the proximal portion only reacted to steroid hormones, the epithelium being cornified by the action of estrogen and mucified in the presence of progesterone and estrogen. The stratified epithelium of the middle portion and the cornified epithelium lining the distal portion remained unaffected by either estrogen or estrogen plus progesterone. The two portions were totally incapable of responding to the hormones.

It seems likely that the proximal portion of vagina is of the Muillerian duct origin, and the distal portion, of the urogenital sinus origin, the shortest middle portion being the transitional part between the two. Through what mechanism the three portions are differentiated in androgenized rats remains an enigma. 
Summary. In highly atrophic ovaries of adult rats given 30 daily injections of testosterone propionate from the day of birth, corpora lutea were produced following injections of gonadotropins. However, the corpora lutea were not functional in intact as well as hypophysectomized androgenized rats, so that injections of estradiol resulted in cornification of the epithelium of the proximal portion of vagina. In contrast, corpora lutea produced in androgenized rats bearing hypophysial autografts under the renal capsule actively secreted progesterone. Consequently, injections of estradiol in such animals caused marked mucification of the epithelium lining the proximal portion of vagina. From these findings, it seems evident that, in androgenized rats, although the secretion of gonadotropins is held in check, the release of luteotropin is still inhibited by the hypothalamus. The epithelia of the middle and distal portions of vagina were stratified and cornified, respectively, regardless of hormonic environments.

\section{References}

Pasteels, J. L.: Arch. Biol., 74, 439 (1963).

Schally, A. V., J. Meites, C. Y. Bowers, and A. Ratner: Proc. Soc. Exp. Biol. and Med., 117, 252 (1964).

Takewaki, K.: Endocrinol. Japon., 11, 1 (1964).

- : Proc. Japan Acad., 41, 310 (1965). 